\title{
Two-Stage Diversity-Based Multiple Description Image Coding
}

\author{
Chunyu Lin, Yao Zhao, Member, IEEE, and Ce Zhu, Senior Member, IEEE
}

\begin{abstract}
In this letter, a diversity-based two-description image coding scheme is firstly presented and analyzed, for which a twostage coding is introduced to facilitate the tuning of central/side distortion tradeoff. By subsampling the central decoded errors from the first stage to constitute the second part for each description, respectively, we show that not only the central distortion but also the side distortion can be reduced with the second stage information. We further generalize the proposed scheme to 4-description coding. Experiment results demonstrate that the proposed scheme outperforms the state-of-the-art techniques in terms of both central and side coding performance.
\end{abstract}

Index Terms-Diversity coding, image compression, multiple description coding.

\section{INTRODUCTION}

I $\mathrm{N}$ the multiple description coding (MDC) approach, one source is encoded into two or more representations (descriptions) which can then be transmitted over separate channels. If only one channel works, the side decoder can reconstruct the source with a certain desired fidelity associated with a so-called side distortion. When more channels work, the reconstruction quality can be enhanced up to the smallest central distortion upon the reception of all descriptions, which is lower bounded by the distortion attainable by single description coding. However, achieving the best central and side coding performance at the same time is shown to be conflicting, and a good tradeoff between central and side coding performance is desired [1].

For image MDC, many approaches have been proposed. Multiple description scalar quantization (MDSQ) is one of the first practical MDC schemes, which is applied to wavelet-based image coding in [2]. A modified MDSQ (MMDSQ) is designed and applied to image coding in [3]. Pairwise correlation transform is presented in [4] and multiple description lattice vector quantization (MDLVQ) based image coding is developed in [5]. All of the above methods exploit some single description (SD) image coders with MDC adaptations to strike a central-side coding tradeoff.

In [6], a novel MD scheme based on diversity is employed for speech coding, and the transform diversity is applied to image

Manuscript received June 02, 2008; revised July 26, 2008. This work was supported in part by the National Natural Science Foundation of China (No. 60776794, No. 90604032), 973 program (No. 2006CB303104), 863 program (No. 2007AA01Z175), PCSIRT (No. IRT0707) and Specialized Research Foundation of BJTU. The associate editor coordinating the review of this manuscript and approving it for publication was Dr. Konstantinos N. Plataniotis.

C. Lin and Y. Zhao are with the Institute of Information Science, Beijing Jiaotong University, Beijing, China (e-mail: yuailian@gmail.com; yzhao@center. njtu.edu.cn).

C. Zhu is with the School of Electrical and Electronic Engineering, Nanyang Technological University, Singapore (e-mail: eczhu@ntu.edu.sg).

Digital Object Identifier 10.1109/LSP.2008.2005044 coding in [7]. For the diversity-based MDC approaches, some existing single description image coding schemes are adopted with little change, which facilitates the easy implementation. More importantly, the central coding performance can still be improved even when the side coder achieves the same best performance as its corresponding single description coder at a rate. However, it becomes difficult to achieve a flexible central-side coding tradeoff with the diversity-based MDC scheme, which may limit its applications.

In this letter, we present a two-stage diversity-based MDC scheme, in which the tradeoffs between central and side coding performance can be tuned easily and flexibly. More importantly, in the side decoder, we find that the side reconstruction quality can be improved by taking into account the subsampled central decoded errors appended to each description in the second stage. Note that in the two-stage MMDSQ [3], the second stage information can only reduce the central distortion but to no avail for side distortion when only one description is received. In contrast, our proposed two-stage diversity-based MDC scheme can help improve not only the central coding performance but also the side performance with the second stage information. Moreover, the proposed scheme can be generalized to $n$-description coding easily. The remainder of this letter is organized as follows. Section II introduces the diversity-based MD image coding scheme with quadtree classification and trellis coded quantization (QTCQ). Then the proposed two-stage diversity-based MDC scheme is presented with analysis and its extension to 4-description coding is also included in Section III. Experimental results are shown in Section IV, and this letter is concluded in the last section.

\section{DIVERSITY-BASED MDC}

In the context of MDC, diversities may be presented as path diversity, time diversity, spatial diversity, and coder diversity [6]. MDC itself can be seen as a path diversity scheme where multiple descriptions can be delivered through different paths. Coding signal and its delayed version or the version of its elementary transformation can form the time or spatial diversity, while adopting different coding schemes for descriptions can lead to coder diversity. In the proposed balanced MDC scheme, the spatial diversity is employed, that is, the diversity is obtained by image shifting/rotating.

\section{A. Diversity-Based Two-Description Image Coding}

A diversity-based two-description image coding scheme is shown in Fig. 1. An image is wavelet-transformed (WT) and is coded by the QTCQ approach [8] to produce description 1. To generate description 2, the image is rotated by 180 degrees and then goes through the same coding process as for the first 


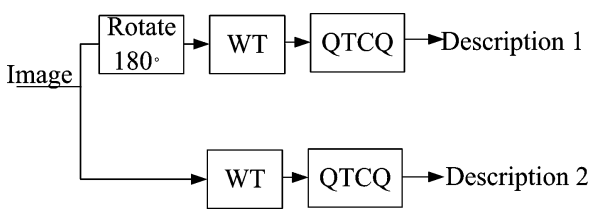

Fig. 1. Diversity-based two-description coding with QTCQ

description. When both the two descriptions are received, they are decoded and averaged to get a better central reconstruction. In view that the wavelet coefficients of the original image and that of the rotated image are different, this diversity can also be considered as transform diversity.

QTCQ has shown its superior performance in image coding [8], which uses the quadtree structure to classify wavelet coefficients and applies TCQ to quantize the classified wavelet coefficients. It is known that quantizing the signal and its elementary transformation version with vector quantizer (VQ) can form diversity [6]. Therefore, using QTCQ can help to further enhance the diversity because the quantization style of TCQ is similar to that of VQ.

\section{B. Distortion Analysis}

For the coding scheme in Fig. 1, assume $\hat{x}_{1}$ and $\hat{x}_{2}$ are the side reconstructed counterparts of an original wavelet coefficient $x$, respectively, as

$$
\begin{aligned}
& \hat{x}_{1}=x+e_{1} \\
& \hat{x}_{2}=x+e_{2}
\end{aligned}
$$

where $e_{1}$ and $e_{2}$ denote the errors generated in the side encoding. The central reconstruction is just the average of the two side ones

$$
\hat{x}_{0}=0.5\left(\hat{x}_{1}+\hat{x}_{2}\right)=x+0.5\left(e_{1}+e_{2}\right) .
$$

In view of the same coding mechanism for the two balanced descriptions, we assume the errors have the same mean of zero and same variance of $\delta^{2}$. Then the side distortion $d_{1}$ and $d_{2}$ are almost the same; therefore, only $d_{1}$ is given, as follows:

$$
d_{1}=E\left[\left(x-\hat{x}_{1}\right)^{2}\right]=E\left[e_{1}^{2}\right]=\delta^{2} .
$$

The central distortion $d_{0}$ is

$$
\begin{aligned}
d_{0} & =E\left[\left(x-\hat{x}_{0}\right)^{2}\right]=E\left[\left(0.5\left(e_{1}+e_{2}\right)\right)^{2}\right] \\
& =E\left[0.25\left(e_{1}^{2}+e_{2}^{2}\right)\right]+0.5 E\left[e_{1} e_{2}\right] \\
& =0.5 \delta^{2}(1+\rho) \leq \delta^{2}
\end{aligned}
$$

where $\rho$ is the correlation coefficient between $e_{1}$ and $e_{2}$. It shows that the central distortion will be lower than the side distortion if $\rho$ is less than 1 . If the error $e_{i}$ of each description is uncorrelated to each other, i.e., $\rho=0$, the central distortion will only be half of the side distortion. This can be translated to a $3 \mathrm{~dB}$ gain in the central distortion over the side distortion. A larger gain can be achieved if the errors of the two descriptions are negatively correlated. We can see that the gap between the central distortion and side distortion depends on the correlation coefficient $\rho$ that is in turn determined by the selected diversity scheme. It is at least not straightforward if not hard as well as

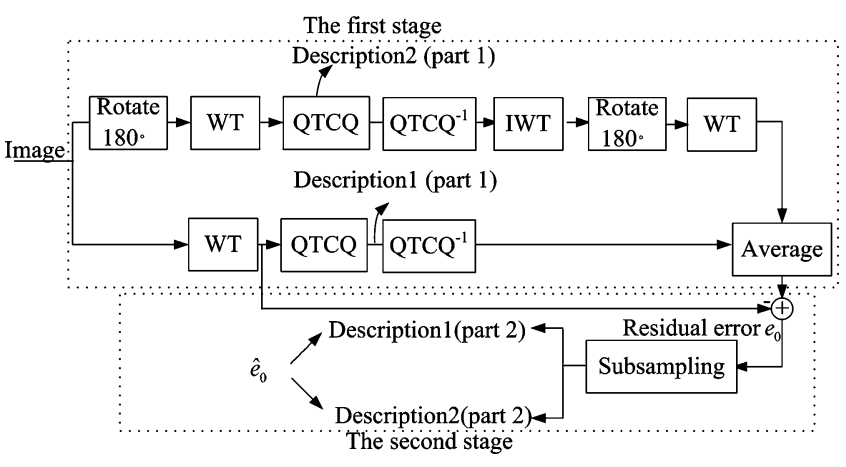

Fig. 2. Proposed two-stage diversity-based MD image coding scheme.

not easily manageable by designing different diversity schemes to change $\rho$, which makes the tuning of central-side distortion tradeoff very troublesome and difficult. Therefore, we consider adopting a second stage based on the scheme in Fig. 1 for an easy and flexible control of the tradeoff.

\section{Proposed Two-Stage Diversity-Based Coding}

\section{A. Proposed 2-Description Coding Scheme}

The proposed two-stage diversity-based 2-description coding scheme is illustrated in Fig. 2. Each description comprises two parts formed in the first and the second stage, respectively. Part 1 is generated as that in Fig. 1, and then the residuals are obtained by subtracting the central (averaged) reconstruction in stage 1 from the original wavelet coefficients. The wavelet residuals are split by subsampling and coded with QTCQ to produce part 2 information for each description.

Note that the MMDSQ [3] also uses a similar two-stage structure. When both descriptions are received, the second stage decoded information (part 2) is added to the average of the description 1 (part 1) and description 2 (part 1) to further enhance the reconstruction quality, which is similar to the MMDSQ central decoding [3]. However, the side decoding of the proposed scheme is much different from that of MMDSQ. In the side decoding of the proposed scheme, when only one description is received, the reconstructed information from part 2 will also be exploited to be added to the part 1 of the received description, which will be shown to help reduce the side distortion in the following. In the MMDSQ [3], however, when only one description is received, the second part information is completely useless, which cannot be used for improving the side decoding and therefore discarded.

\section{B. Side Distortion Analysis}

The residual errors between the original wavelet coefficients and the averaged reconstructions from part 1 information can be represented as

$$
e_{0}=x-\hat{x}_{0}=-0.5\left(e_{1}+e_{2}\right) .
$$

The errors are split with subsampling and then coded by QTCQ to form the second part for each description. For simplicity, we assume the odd/even coefficient subsampling way. For the balanced descriptions, only description 1 is considered, while the other can be analyzed similarly. In the decoding end, the odd 


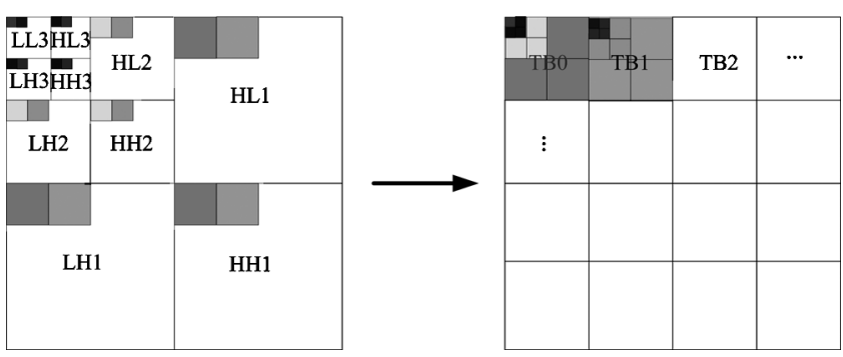

Fig. 3. Subsampling method.

samples of the second part are added to the first part of description 1 . Then description 1 becomes

$$
\hat{x}_{1}^{\prime}= \begin{cases}x+e_{1}+\hat{e}_{0}, & \text { for odd coefficients } \\ x+e_{1}, & \text { for even coefficients }\end{cases}
$$

where $\hat{e}_{0}$ denotes the reconstructed counterpart of $e_{0}$. We make the asymptotic analysis of adding the decoded odd part of central residuals to the side reconstruction, that is, $\hat{e}_{0}$ approximates $e_{0}$. Substituting $e_{0}$ with that in (6), we can obtain the side distortion for description 1 as

$$
\begin{aligned}
d_{1}^{\prime} & =E\left[\left(x-\hat{x}_{1}^{\prime}\right)^{2}\right]=0.5 E\left[\left(e_{1}\right)^{2}\right]+0.5 E\left[\left(e_{1}+e_{0}\right)^{2}\right] \\
& =0.5 \delta^{2}+0.5 E\left[\left(0.5\left(e_{1}-e_{2}\right)\right)^{2}\right] \\
& =0.5 \delta^{2}+0.25 \delta^{2}(1-\rho) \leq \delta^{2} .
\end{aligned}
$$

The side distortion for description 2 can also be obtained as (8) in the balanced coding. The equality of the above formula is valid only when $\rho=-1$, which means no reduction of side distortion. In this case, however, the central distortion will be zero which can be seen from (5), thus no residual error and no bit cost in the second stage. For $0 \leq \rho<1$ which is observed in our experiments, the second stage information can help reduce the side distortion from $\delta^{2}$ to the range of $0.5 \delta^{2} \sim 0.75 \delta^{2}$.

In view of the strong correlation in wavelet subbands, we consider subsampling the coefficients in a way of odd/even wavelet tree block to facilitate the following QTCQ for a better compression. Taking a 3-level wavelet transform as an example, the subsampling approach is shown in Fig. 3, which is like the zerotree structure. That is, the wavelet coefficients in different subbands corresponding to the same spatial location are grouped to form a tree block, and then these blocks are subsampled in an odd/even way.

\section{Flexible Tradeoff Control}

In the proposed two-stage scheme, the tradeoff between the central and side distortion is tuned by the bit rate allocation in the first and the second stage. When the channel condition is bad, less or no bits will be allocated to the second stage, to favor side decoding. In the case of skipping the second stage, the side coding performance may be maximized, which is equivalent to a corresponding SD scheme at the same side coding rate, and the central performance can still be enhanced to some extent due to the diversity. Note that in the same case of skipping the second stage in MMDSQ (degraded to normal MDSQ), if the side coding performance is maximized, the central performance will be the same as that without any further improvement. When

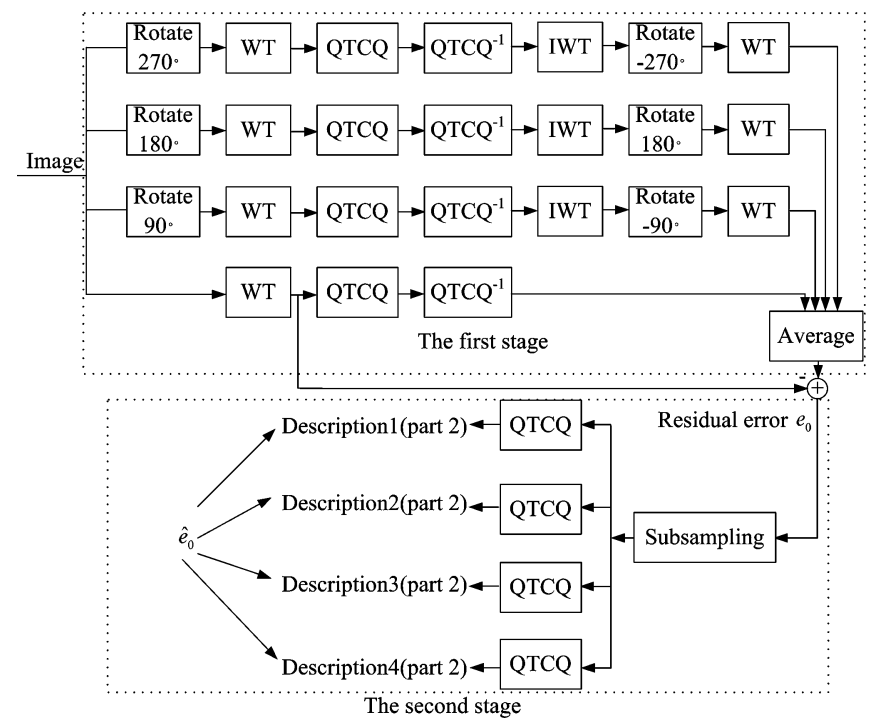

Fig. 4. Proposed 4-description image coding.

the network condition is good, more or even all the bits are assigned to the second part to favor central decoding. When allocating all the bits to the second stage, each of the bitstreams contains half of the information and the central coding performance will be improved significantly. In between for the bit allocation, varying central-side coding tradeoffs can be obtained.

\section{Extension to 4-Description Coding}

The proposed scheme can be generalized to $n$-description scheme conveniently. As an example, 4-description coding is illustrated in Fig. 4. The residual error is subsampled in the similar tree block way. In the decoding end, if two or more descriptions are received, the first parts are decoded and averaged before adding the second decoded parts.

\section{EXPERIMENTAL RESULTS}

\section{A. The 2-Description Coding}

For the sake of comparison, we also give the performance of MDSQ-based wavelet coder by Servetto et al. [2], modified MDSQ based on Tarp filter image coder with classification for embedding (MMDSQ + TCE) [3], the transform and data fusion scheme [7], the JPEG2000-based MD approach [9], and feature-oriented MDC (FOMDC) [10]. All of the five methods including ours make use of wavelet transform in their coding scheme. A six-level wavelet decomposition is applied with the Daubechies 9/7 filters and 8-state TCQ is used in the QTCQ scheme. The tested rate is selected to be 0.5 bpp per description. The central and side PSNR performance for "Lena" (of size $512 \times 512$ ) is shown in Fig. 5. Due to the page limit, the result on another image "Barbara" is not included which exhibits the similar performance.

It can be seen from the figure that the proposed scheme achieves the best central-side distortion performance at the rate of $0.5 \mathrm{bpp}$ per description. Note that the transform and data fusion scheme [7] has only the first stage structure of the proposed scheme, thus, as mentioned before, making it difficult to achieve varying tradeoff points, where only two tradeoff 


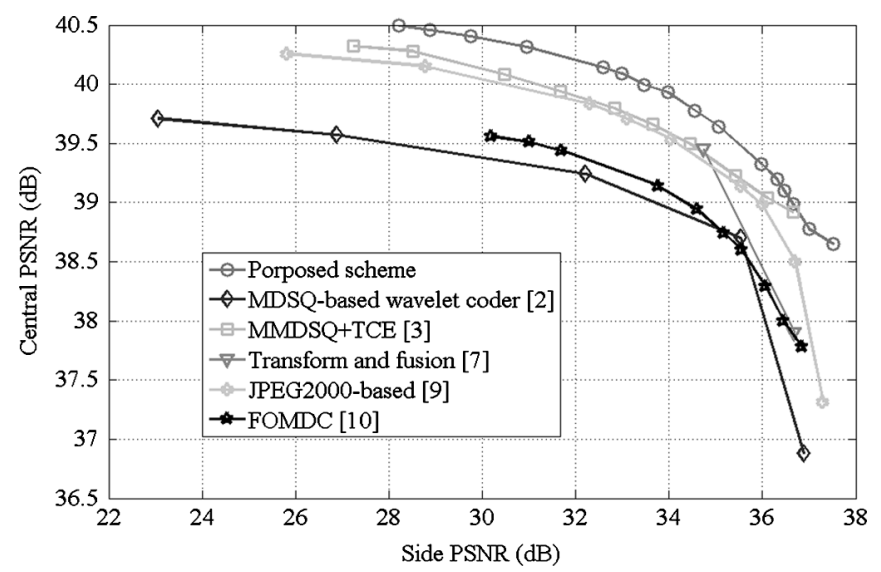

Fig. 5. Comparison of central-side PSNR curves at $0.5 \mathrm{bpp} /$ description for "Lena."

points are obtained. With our two-stage scheme, the tradeoff points can be obtained conveniently and flexibly by changing the bit allocation in the two stages, where many points are indicated in the figure. Furthermore, we can see that as side PSNR increases with more redundancy introduced, the central PSNR will drop dramatically to be close to the side PSNR for the schemes like in [2], [9], and [10], whereas in our proposed scheme with few bits for the second stage, the central PSNR is still better than the side PSNR over 1-2 dB. The better central-side coding performance is due to the high-performance QTCQ coding as well as our proposed two-stage MDC scheme. For example, in the single description coding for "Lena," the QTCQ coder can obtain $40.75 \mathrm{~dB}$ of PSNR at $1 \mathrm{bpp}$ which outperforms JPEG-2000 around $0.3 \mathrm{~dB}$, while our proposed MDC scheme can achieve about $1 \mathrm{~dB}$ gain in average over the JPEG-2000 based MDC in side distortion performance given a central distortion at the total rate of $1 \mathrm{bpp}$, as can be seen in Fig. 5. The complexity for the proposed scheme increases since two stages are involved. Running on a 2.99-GHz Pentium computer with Windows XP Professional, it takes around 2.23 $\mathrm{s}$ to encode a $512 \times 512$ image into two descriptions at 0.5 bpp/description, and $1.81 \mathrm{~s}$ to decode two side descriptions and one central description, without any program optimization. The JPEG-2000-based MDC scheme, however, requires longer running time on the same platform, i.e., about $6 \mathrm{~s}$ for encoding and $3 \mathrm{~s}$ for decoding.

\section{B. The 4-Description Coding}

The 4-description coding results are shown in Fig. 6 to compare the proposed scheme against the JPEG2000-based MD scheme [11] for the image "Lena" at the total target rate of $2.2 \mathrm{bpp}$ and $2.0 \mathrm{bpp}$ with two redundancy rates of $0.3 \mathrm{bpp}$ and 0.6 bpp, respectively. For the JPEG2000-based scheme, we use the software available in the website [11], which, to our observation, cannot always assure an accurate total bit rate for a given redundancy rate. By adjusting the quantization in the two stages, our two-stage coder is easy to tune to produce the same total bit rate and redundancy rate as that of the JPEG2000-based scheme. In our scheme, the rate of the first stage is considered as the redundancy rate. It can be seen that the proposed scheme consistently and significantly outperforms the JPEG2000-based

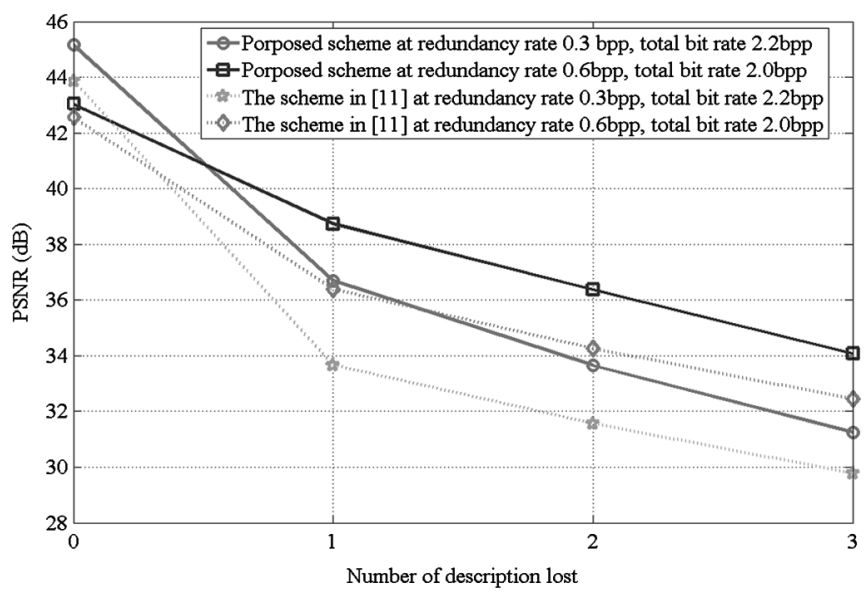

Fig. 6. Comparison of 4-description coding for "Lena."

scheme at the two redundancy rates in the case of losing one or more descriptions.

\section{CONCLUSION}

A two-stage diversity-based MD scheme has been proposed for a flexible central/side distortion tradeoff. More importantly, the second stage information for each description obtained by subsampling the central residual errors from the first stage has been shown to improve the side coding performance in addition to the central performance. The scheme can be extended to n-description coding in a convenient way. The experiment results on image coding have shown the superior central/side coding performance of the proposed scheme over a few state-of-the-art MD approaches.

\section{REFERENCES}

[1] V. K. Goyal, "Multiple description coding: Compression meets the network," IEEE Signal Process. Mag., vol. 18, no. 5, pp. 74-93, Sep. 2001.

[2] S. D. Servetto et al., "Multiple description wavelet based image coding," IEEE Trans. Image Process., vol. 9 , no. 5 , pp. 813-826, May 2000.

[3] C. Tian and S. S. Hemami, "A new class of multiple description scalar quantizer and its application to image coding," IEEE Signal Process. Lett., vol. 12, no. 4, pp. 329-332, Apr. 2005.

[4] Y. Wang, M. T. Orchard, V. Vaishampayan, and A. R. Reibman, "Multiple description coding using pairwise correlating transforms," IEEE Trans. Image Process., vol. 10, no. 3, pp. 351-366, Mar. 2001.

[5] H. Bai, C. Zhu, and Y. Zhao, "Optimized multiple description lattice vector quantization for wavelet image coding," IEEE Trans. Circuits Syst. Video Technol., vol. 17, no. 7, pp. 912-917, Jul. 2007.

[6] X. Zhong and B.-H. Juang, "Multiple description speech coding with diversities," in Proc. IEEE Int. Conf. Acoustics, Speech, and Signal Processing, Orlando, FL, 2002, vol. 1, pp. 177-180.

[7] S. Tian and P. K. Rajan, "Multiple description coding using transforms and data fusion," in Proc. Int. Conf. Information Technology: Coding and Computing, 2005, vol. 1, pp. 85-90.

[8] B. A. Banister and T. R. Fischer, "Quadtree classification and TCQ image coding," IEEE Trans. Circuits Syst. Video Technol., vol. 11, no. 1, pp. 3-8, Jan. 2001.

[9] T. Tillo and G. Olmo, "A novel multiple description coding scheme compatible with the JPEG2000 decoder," IEEE Signal Process. Lett., vol. 11, no. 11, pp. 908-911, Nov. 2004.

[10] Y. Liu and S. Oraintara, "Feature-oriented multiple description wavelet-based image coding," IEEE Trans. Image Process., vol. 16, no. 1, pp. 121-131, Jan. 2007.

[11] E. Baccaglini, T. Tillo, and G. Olmo, "A flexible R-D-based multiple description scheme for JPEG 2000," IEEE Signal Process. Lett., vol. 14, no. 3, pp. 197-200, Mar. 2007. 